\title{
Low-power Environmental Monitoring System for ZigBee Wireless Sensor Network
}

\author{
Tareq Alhmiedat ${ }^{1}$ \\ ${ }^{1}$ Department of Information Technology, Tabuk University \\ Tabuk, 71421, Saudi Arabia \\ [e-mail: t.alhmiedat@ut.edu.sa] \\ *Corresponding author: Tareq Alhmiedat
}

Received February 1, 2017; revised March 18, 2017; revised April 11, 2017; accepted May 25, 2017; published October 31, 2017

\begin{abstract}
Environmental monitoring systems using Wireless Sensor Networks (WSNs) face the challenge of high power consumption, due to the high levels of multi-hop data communication involved. In order to overcome the issue of fast energy depletion, a proof-of-concept implementation proves that adopting a clustering algorithm in environmental monitoring applications will significantly reduce the total power consumption for environment sensor nodes. In this paper, an energy-efficient WSN-based environmental monitoring system is proposed and implemented, using eight sensor nodes deployed over an area of $1 \mathrm{~km}^{2}$, which took place in the city of Tabuk in Saudi Arabia. The effectiveness of the proposed environmental monitoring system has been demonstrated through adopting a number of real experimental studies.
\end{abstract}

Keywords: Wireless Sensor Network, ZigBee, environmental monitoring, clustering algorithms, energy-aware design. 


\section{Introduction}

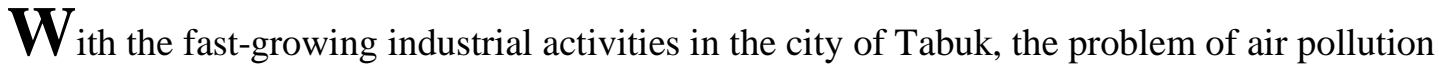
has become a major concern for the health of the population. To improve people's health and safety, it is very useful to observe air quality. Headaches, nausea, eye and throat irritations are common symptoms of the so-called sick building syndrome.

The main intention of environmental monitoring is not only to gather data from a number of locations, but also to provide the information required by scientists, planners and policy-makers, to enable them to make decisions about managing and improving the environment, in addition to presenting helpful information to end-users. Considerable effort is made to improve the air quality in both indoor and outdoor environments.

Wireless Sensor Networks (WSNs) are currently an active area of research due to their wide range of applications, which include military, medical, environmental monitoring, safety and civilian contexts [1-3].

A sensor network is a collection of sensor nodes that are low in cost and have a short communication range, distributed over the area of interest. A sensor node consists of four subsystems, as depicted in Fig. 1. The processing subunit is responsible for processing the data captured from sensor nodes before they are transmitted to the base station. The sensing subunit is a device that produces a measurable response to a change in a physical condition such as humidity or temperature. The communication subunit, on the other hand, includes a short radio range used to communicate with neighbouring nodes. Finally, the power supply subunit contains the battery source to fuel the aforementioned units [4].

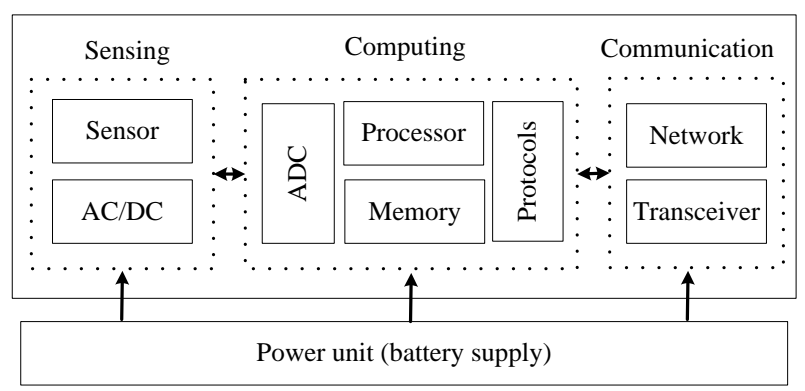

Fig. 1. Sensor node architecture

Habitat and environmental monitoring represent an important category of sensor network applications. Recent advances in low-power wireless network technology have created the technical conditions to build multifunctional tiny sensor devices, which can be used to sense and observe physical phenomena. Many environmental monitoring examples of WSNs have already been presented in the literature and developed for different purposes. Fig. 2 presents the main concept of an environmental monitoring system using WSNs. 


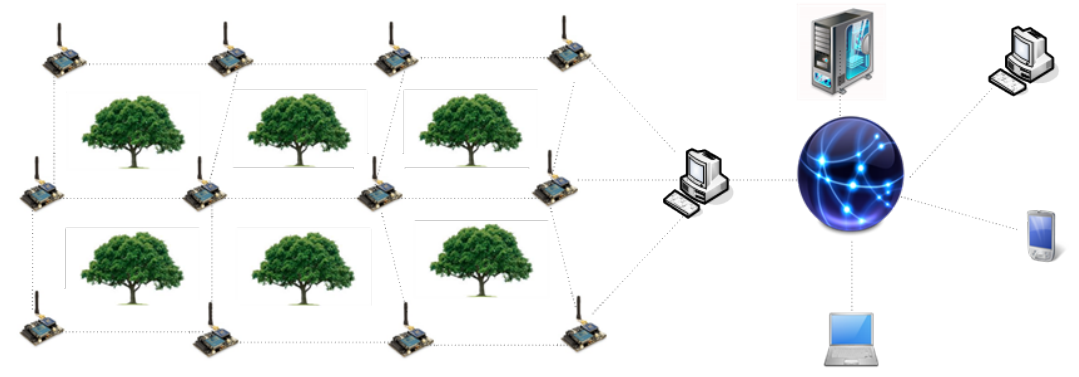

Fig. 2. The concept of real-time environmental monitoring using WSNs

The deployment of WSNs offers an alternative solution in the form of a large number of disposable sensor nodes scattered over an area of interest. The advantages of WSNs in monitoring pollution are flexibility in their deployment and savings in time and money by avoiding power, wiring, and the related infrastructure. A key concern in such networks is energy efficiency, as gas sensors are energy-hungry. In addition, sensor nodes may operate unattended for several months to a few years on a battery power supply. Researchers have developed many protocols mainly designed for WSNs [5-7], where energy consumption is an essential consideration, and attention has been given to the routing protocols and data-gathering algorithms [8,9], as they might differ from traditional networks.

Data gathering in WSNs is an essential task, where various gathering systems have been proposed recently, driven by the requirement to achieve low energy consumption with the best network performance. One of the most energy-efficient data-gathering approaches in WSNs is clustering. Cluster-based protocols are well-known techniques that enable the operation of a WSN to be highly energy-efficient. The basic principle of clustering is to organize sensor nodes into groups, called clusters, where each cluster selects a cluster-head $(\mathrm{CH})$ that has the responsibility of collecting data from other sensor nodes in its cluster, aggregating it and then forwarding the compact information to the sink node. Cluster-based protocols reduce the amount of data transferred within the network. Many clustering protocols have been proposed recently with the objective of maximizing the network lifetime, such as LEACH [7], Fuzzy C-Means [9], LEACH-C [10], PEGASIS [11], and P-SEP [12]. However, most of the existing approaches were validated through simulation studies [8-11]. In addition, some approaches require the transmission of a large number of packets to the nodes located in the vicinity of the sink node, which rapidly depletes their energy.

Therefore, in order to develop the long-lasting operation of WSN-based environmental monitoring applications, this paper addresses the area of data gathering for WSN-based environmental monitoring systems, through real experiments. The main contributions of this paper are:

a. A real-time monitoring system is proposed and implemented to monitor the significant pollutants in the outdoor environments.

b. The minimum energy consumption has been achieved, through adopting an energy management system at the sensor node level and the network level.

c. A new energy-efficient clustering algorithm has been proposed, where sensor nodes are grouped and a $\mathrm{CH}$ is elected based on a novel clustering function.

d. Unlike the existing systems, which mainly focus on simulation experiments, the clustering system has been validated through XBee sensor nodes. 
The remainder of this article is organized as follows: Section 2 reviews the existing relevant works, before the system model is presented and discussed in Section 3. Section 4 presents the hardware architecture, which is implemented to assess the efficiency of the proposed system. Results obtained from real experiments are discussed and analysed in Section 5. Finally, Section 6 presents a conclusion and proposes future work.

\section{Related Work}

Many research groups are working on the design and development of WSN environmental monitoring systems, where each solution differs in the type of sensors used, communication range and in the achieved energy optimization. The various environmental monitoring systems implemented recently are summarized in [13]. In this section, the existing monitoring systems are categorized, based on the sampling frequency rate, into three main categories: periodic-based, event-based and query-based sampling.

Firstly, periodic-based sampling systems are considered. Through this category, the collected data from on-board sensors are frequently transmitted to the base station every $t$ period of time, where $t$ is determined in advance. Several existing environmental monitoring systems are periodic-based [14-32]. However, periodic-based systems are not able to capture events which may occur irregularly, such as fire, as they do not provide early detection.

Secondly, event-based sampling systems are considered. This category includes transmitting the sensed data to a base station as soon as a certain event has occurred (for instance, when the level of a certain gas exceeds a predefined threshold value) [33, 34]. Event-based systems reduce the amount of information exchanged between sensors, controls and actuators. This reduction extends the sensor node's lifetime, reduces the computational load in embedded devices and may also cut down the network bandwidth.

Thirdly, query-based sampling systems are considered. Sensor nodes gather data only upon receipt of a query from the sink node [22, 24, 33, 34, 35]. This category is useful for managing the sensor node's resources, such as in sense communication.

Through investigating the existing WSN-based environmental monitoring systems, researchers mainly focused on three significant issues when developing such applications: energy, scalability and cost.

Environmental monitoring systems are considered to have high energy consumption, since transceiver modules and gas sensors are energy-hungry. Energy is critical for long-lasting operation, especially when the system is required to monitor remote and hostile environments. Data aggregation [19], clustering methods [6, 7, 10], cross-layer protocols [20] and power storage devices $[19,21]$ are presented as possible solutions to improve the sensor nodes' lifetime in environmental monitoring applications.

Clustering is an important mechanism in large WSNs for obtaining scalability and energy-saving and it offers better network performance. One well-known clustering system, called LEACH [7], consists of two phases: the setup phase and the data transmission phase. In the setup phase, the formation of the clusters is carried out. During the transmission phase, sensing information is acquired by sensor nodes and transferred to the base station. LEACH-centralized (LEACH-C), an improvement of LEACH, employs a centralized clustering mechanism to form the clusters [10]. LEACH-C disperses the CHs through the network, which produces better clusters and thus improves the network performance. Authors of [8] have proposed a fuzzy logic-based clustering approach with an energy-periodic mechanism for LEACH through the selection of CHs in order to improve the network lifetime 
further. Other protocols use clustering algorithms such as K-means [8] and FCM [9] to achieve a better formation of clusters. P-SEP [12] is a modified stable election protocol to prolong the stable period of Fog-supported sensor networks by maintaining balanced energy consumption. P-SEP offers three functions: it enables uniform distribution, a new $\mathrm{CH}$-selection policy and prolongs the time interval of the system.

WSN can accommodate thousands of nodes in environmental monitoring applications. Therefore, it is essential to prove that the available theoretical solutions are suited to large real WSNs. Several works $[14,15,25,27,30,32]$ have presented the results of experiments using a small number of sensor nodes.

In environmental monitoring applications, sensor networks are meant to be deployed in thousands of nodes to achieve wider coverage. Therefore, the sensor nodes must be low in cost. The available wireless sensor platforms on the market are relatively expensive [21, 22, 30, 33, $34,35]$. Hence, producing a cheaper sensor node platform is a requirement. On the other hand, simulation experiments are unable to capture the complexity of outdoor environments [7-10], which affects the WSN functionalities. Therefore, real-world deployments and experiments are necessary to study and analyse their harsh conditions. In this research, the author investigates the use of WSNs for monitoring air pollution monitoring in the city of Tabuk using real experiments, in which an energy-efficient environmental monitoring system has been designed and implemented through the adoption of a new clustering algorithm, using inexpensive sensor nodes, to minimize the total amount of energy required for such applications. Table 1 presents a comparison between the various deployment systems in the literature, in terms of sampling rate (periodic, event and query), lifetime, cost and scalability.

Table 1. A comparison between various deployment systems in the literature

\begin{tabular}{|c|c|c|c|c|c|c|c|c|}
\hline \multirow{2}{*}{ Reference } & \multirow{2}{*}{ Size } & \multicolumn{3}{|c|}{ Sampling rate function } & \multicolumn{3}{|c|}{ Considered parameters } & \multirow{2}{*}{$\begin{array}{c}\text { Communication } \\
\text { platform }\end{array}$} \\
\hline & & Periodic & Event & Query & Lifetime & Cost & Scale & \\
\hline [14] & 1 & $\sqrt{ }$ & - & - & $\sqrt{ }$ & $\sqrt{ }$ & - & Mica 5121 \\
\hline [15] & 2 & $V$ & - & - & - & - & $\sqrt{ }$ & XBee series 2 \\
\hline [16] & 5 & $\sqrt{ }$ & - & - & - & - & $\sqrt{ }$ & XBee series 1 \\
\hline [17] & 42 & $\sqrt{ }$ & - & - & - & - & $\sqrt{ }$ & Micaz \\
\hline [18] & 5 & $\sqrt{ }$ & - & - & - & $\sqrt{ }$ & $\sqrt{ }$ & iAQ-2000 \\
\hline [19] & 4 & $V$ & - & $\sqrt{ }$ & $\sqrt{ }$ & - & - & XBee series 1 \\
\hline [20] & 36 & $\sqrt{ }$ & - & - & $\sqrt{ }$ & $\sqrt{ }$ & - & Jennic JN5184 \\
\hline [21] & 17 & $\sqrt{ }$ & - & - & - & - & - & Shockfish Tiny node \\
\hline$[22]$ & 25 & $\sqrt{ }$ & - & $\sqrt{ }$ & - & - & - & CC2431 \\
\hline [23] & 7 & $\sqrt{ }$ & - & - & $\sqrt{ }$ & - & - & XBee Pro \\
\hline [24] & 8 & $\sqrt{ }$ & - & $\sqrt{ }$ & $\sqrt{ }$ & - & - & Crossbow IRIS \\
\hline [25] & 3 & $\checkmark$ & - & - & $\sqrt{ }$ & - & - & XBee series 2 \\
\hline [26] & 32 & $\sqrt{ }$ & - & - & $\checkmark$ & - & $\sqrt{ }$ & Berkeley motes \\
\hline [27] & 3 & $\sqrt{ }$ & - & - & $\sqrt{ }$ & - & $\sqrt{ }$ & XBee series 1 \\
\hline [28] & 8 & $\sqrt{ }$ & - & - & - & - & $\sqrt{ }$ & ARM9 S3C2410 \\
\hline [29] & 3 & $\sqrt{ }$ & - & - & $\sqrt{ }$ & - & $\sqrt{ }$ & NI WSN-3202 \\
\hline [30] & 2 & $V$ & - & - & - & - & $\sqrt{ }$ & XBee series 1 \\
\hline
\end{tabular}




\begin{tabular}{|c|c|c|c|c|c|c|c|c|}
\hline [31] & 3 & $\sqrt{ }$ & - & - & - & - & $\sqrt{ }$ & XBee series 2 \\
\hline [32] & 2 & $\sqrt{ }$ & - & - & - & $\sqrt{ }$ & $\sqrt{ }$ & XBee series 1 \\
\hline [33] & 4 & - & $\sqrt{ }$ & $\sqrt{ }$ & - & - & $\sqrt{ }$ & Duganit WL-2410 \\
\hline [34] & 6 & - & $\sqrt{ }$ & $\sqrt{ }$ & - & - & $\sqrt{ }$ & GPRS modem \\
\hline [35] & 4 & - & - & $\sqrt{ }$ & $\sqrt{ }$ & - & - & GPRS modem \\
\hline [this work] & 8 & $\sqrt{ }$ & $\sqrt{ }$ & $\sqrt{ }$ & $\sqrt{ }$ & $\sqrt{ }$ & $\sqrt{ }$ & XBee series 2 \\
\hline
\end{tabular}

* This $\sqrt{ }$ means that the corresponding metric is considered. The - means the metric is not considered.

\section{System Design}

A tiered architecture system that includes two layers has been designed and implemented, as presented in Fig. 3. The lowest layer consists of scattered sensor nodes that sense the environmental gases and transmit their data through the ZigBee network to the sink node (base station), whereas the top layer includes the network layer that groups the sensor nodes into clusters in order to achieve the lowest possible energy consumption. This section discusses the sensor node level and network level.

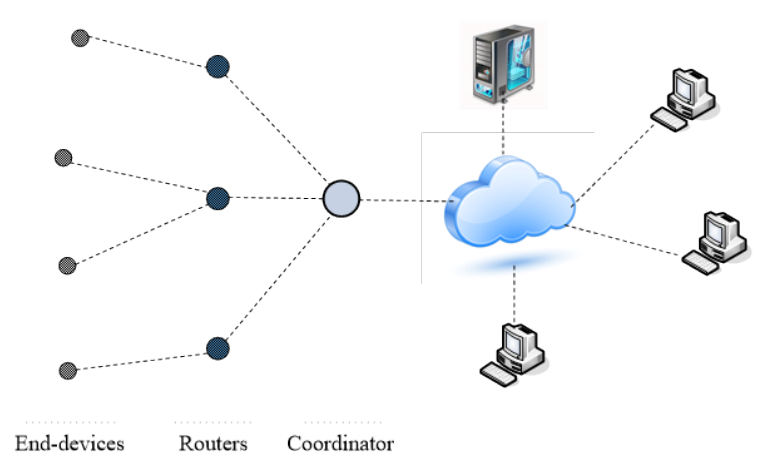

Fig. 3. Sensor network architecture for environmental monitoring applications

\subsection{Sensor Node Level}

The lowest level of the sensing application is the sensor nodes, which perform general purpose computing and networking, in addition to application-specific sensing. WSNs are often built using small and inexpensive individual sensor nodes. Each sensor node collects environmental data primarily about its immediate surroundings and transmits these to the sink node, where the sink node is responsible for transmitting sensed data to the end-user. The sensor node architecture is presented in Fig. 4, where the sensor array consists of two air pollution sensors ( $\mathrm{CO}$ and $\mathrm{CO} 2$ ), in addition to a temperature sensor. 


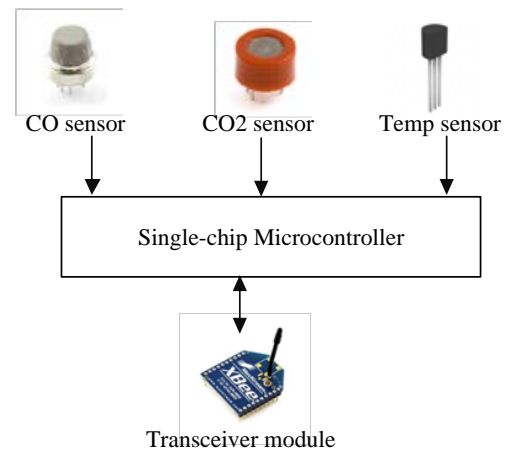

Fig. 4. Sensor node architecture

In order to minimize the energy consumption at the sensor node level, an energy-efficient system has been adopted, which consists of two subsystems: an adaptive sampling subsystem and a power management subsystem. The former possesses the main characteristics of event, periodic, and query-based sampling systems, where each sensor node may transmit its gathered data as soon as a certain event has occurred, every $t$ period of time if no event has occurred during the period $t$, or based on a user's request.

Usually, sensor nodes are designed with light energy-consumption sensors (e.g., light, temperature, humidity and pressure). However, gas sensors consume higher levels of energy than any other components on the sensor node, since gas sensors need to be heated to measure the level of a certain gas. Therefore, to minimize the amount of energy required for such sensors, an interface circuit has been designed to control the state for such sensors and thus minimize the energy consumption.

On the other hand, radio transceivers consume a large amount of energy when transmitting, receiving or listening. Therefore, to obtain efficient power consumption by individual sensor nodes, a power management system is adopted, in which sensor nodes may enter a sleep mode if no request is received from neighbouring or sink nodes, or if no significant data need to be sent from the sensor nodes. Fig. 5 presents the flowchart that illustrates the sleep time management function at the sensor node level. Both microcontroller and transmission modules enter sleep mode in order to minimize the energy consumption. Algorithm 1 presents the code runs at the sensor node level.

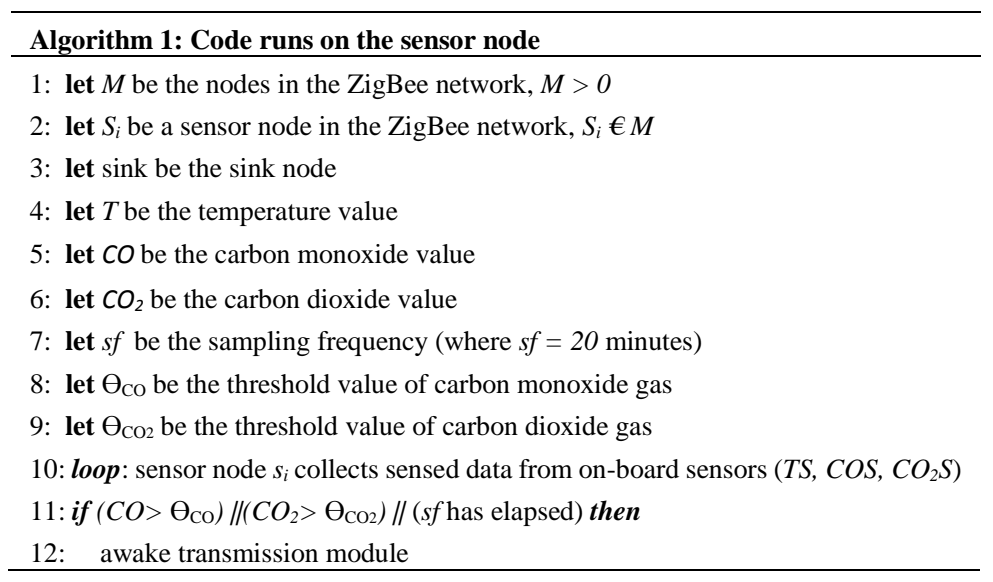



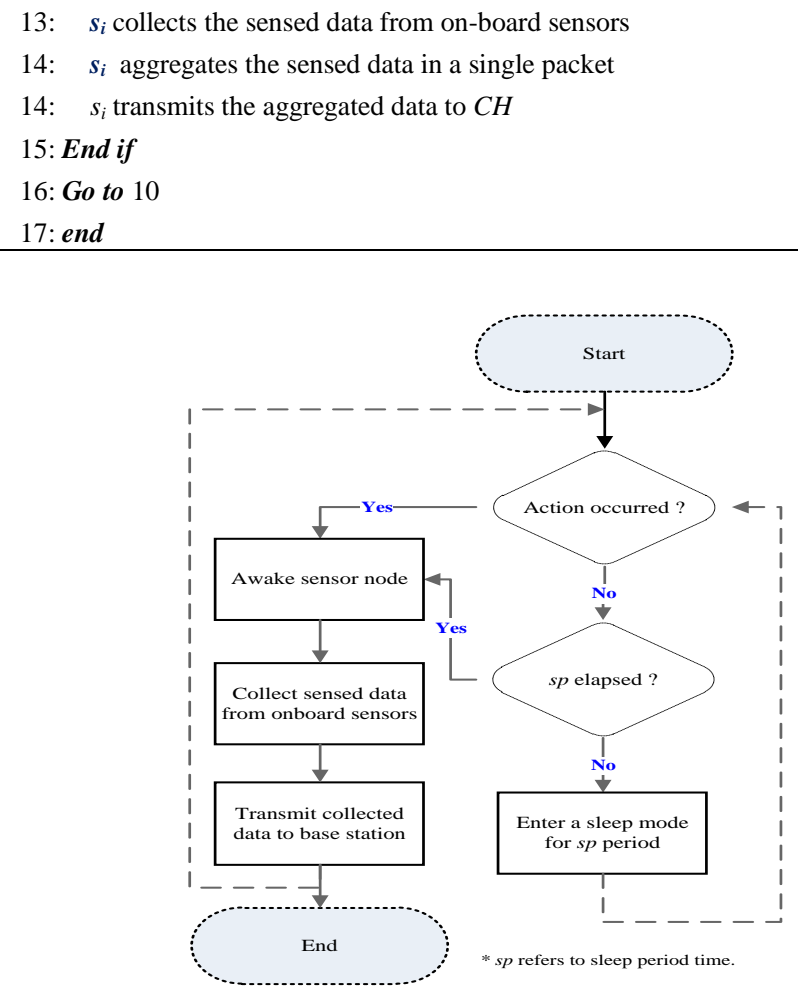

Fig. 5. Flowchart of the hybrid sampling system

\subsection{Network Level}

The top layer is the network layer, presented in Fig. 6, which consists of a number of sensor nodes distributed over an area of interest. In environmental monitoring applications, sensor nodes are required to operate unattended for several months to few years. Since different nodes in the network have different functionalities, they may also have different levels of energy consumption. For instance, nodes near the sink node may need to sense the environmental characteristics, in addition to forwarding packets from neighbouring nodes to the sink node. Therefore, their energy will drain first, so the sensor network will be inaccessible. The designed sensor node runs on a 9V battery with 2.5 amperes, hence, employing an energy-efficient data-gathering algorithm is a requirement. A new clustering algorithm is introduced in this section, which aims to reduce the amount of energy required to collect the sensed data from distributed sensor nodes. 


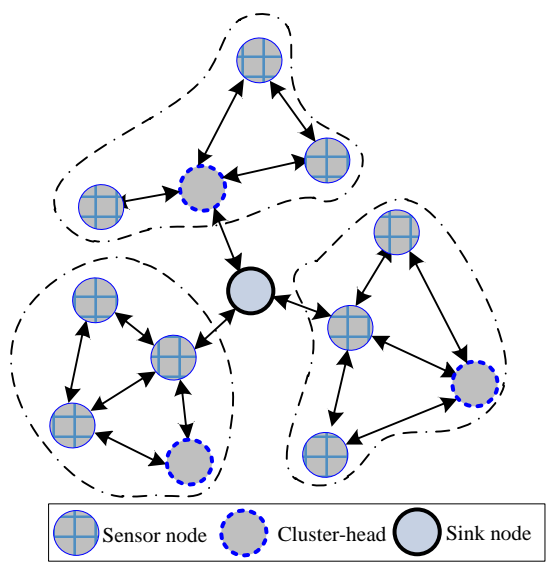

Fig. 6. Network level architecture

The network level is divided into two phases: cluster formation and $\mathrm{CH}$ election. During the cluster formation phase, the sensor nodes form the clusters, whereas during the $\mathrm{CH}$ election phase an appropriate $\mathrm{CH}$ is chosen to collect and aggregate sensed data from the sensor nodes in a certain cluster. The cluster formation method implemented in this work is the same as the one introduced in [6].

As soon as clusters have been formed, each cluster assigns a $\mathrm{CH}$, where the $\mathrm{CH}$ has diverse roles, including: collecting the sensed data from neighbouring nodes in the same cluster, maintaining the optimal sampling frequency rate, managing an idle sleep state period for itself and for the sensor nodes in its cluster, and electing a new $\mathrm{CH}$ for the next round.

In order to balance the remaining energy for all sensor nodes in a certain cluster, data gathering is divided into rounds. In each round, a single $\mathrm{CH}$ is elected based on three factors: the type of sensor node (end-node or router node), the remaining energy of the sensor node, and the obtained signal strength value between the sink node and the elected $\mathrm{CH}$. The cluster function $(C F)$ is presented in Equation 1, which includes the total values of the remaining energy $(E)$, type of sensor node $(T)$ and received signal strength $(S)$. The current $\mathrm{CH}$ has the role of collecting $C F$ values from neighbouring nodes in its cluster and finding the sensor node with the best $C F$ value. The sensor node with the best $C F$ value will play the role of the $\mathrm{CH}$ for the next round. Algorithm 2 presents the code runs on the $\mathrm{CH}$ node.

$$
C F=E+T+S
$$

The remaining energy function $E_{s i}$ for a sensor node si is presented in Equation 2, where this function aims to balance the remaining energy for sensor nodes in a certain cluster.

$$
E_{s_{i}}=\frac{1}{R_{s_{i}}}
$$

where $R_{s i}$ is the remaining energy for sensor node $s_{i}$.

Sensor nodes placed close to a sink node will drain their energy first, since they participate in sensing the environment and forwarding sensed data to the sink node, so they cannot enter sleep mode. Therefore, to minimize the energy required by such nodes, sensor nodes are divided into two types: router nodes and end-nodes. The sensor node which does not participate in routing is called the end-node, whereas the sensor node which is on the trajectory of the end-nodes and participates in routing is called a router node. 


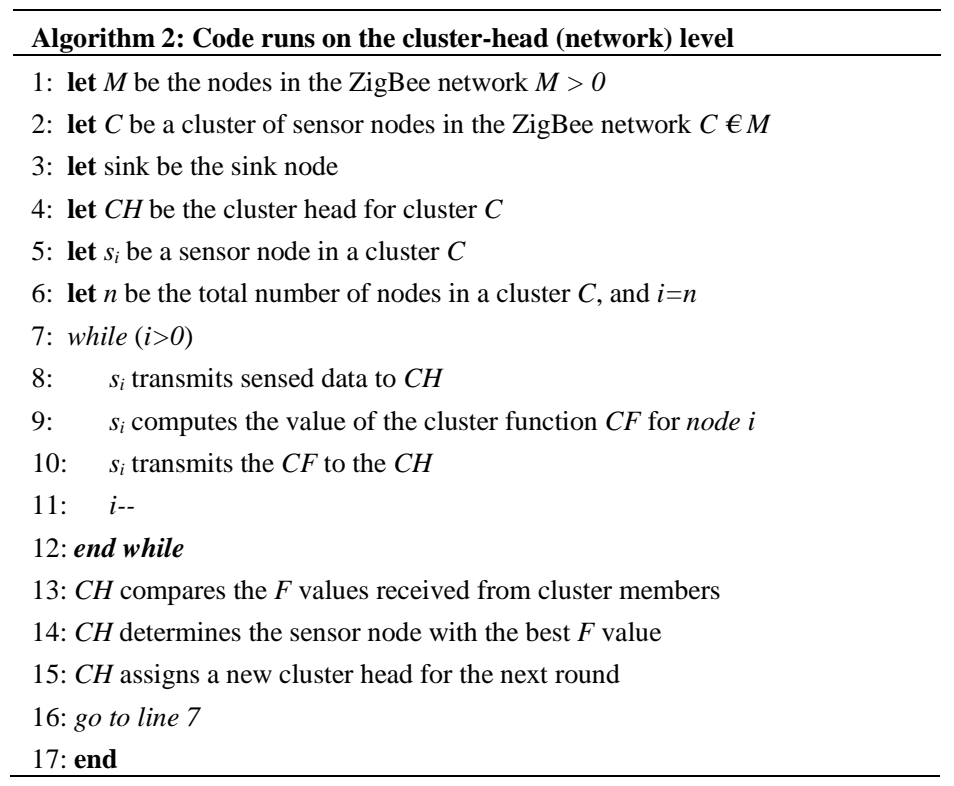

To discuss the effect on energy consumption of the sensor node's type, two experiments have been conducted. Each experiment consists of six sensor nodes (a sink node, a single $\mathrm{CH}$, and four child-nodes), as presented in Fig. 7, where both experiments last for 10 minutes. In the first experiment (Fig. 7-A), end-nodes transmit a single packet every 30 seconds to the $\mathrm{CH}$ (the node which has the shortest distance to the sink node, i.e., node 1), where the $\mathrm{CH}$ is a router node (because it is on the trajectory of the end-nodes). In the second experiment (Fig. 7-B), an end-node (for instance, node 4) plays the role of $\mathrm{CH}$.

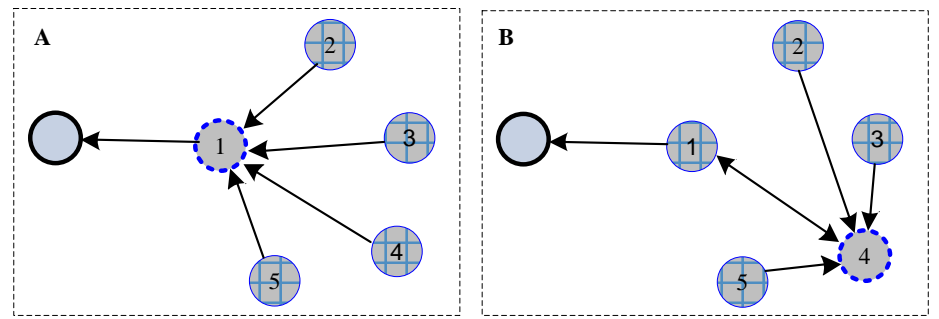

Fig. 7. Sensor deployment in two scenarios, A and B

Apparently, as presented in Fig. 7-A (experiment A), sensor nodes 2, 3, 4 and 5 transmit the sensed data to the $\mathrm{CH}$ (node 1). Therefore, the $\mathrm{CH}$ (node 1) has the role of gathering the sensed data from sensor nodes in its cluster and transmitting the collected data to the sink node. Conversely, in the second experiment depicted in Fig. 7-B, sensor nodes 1, 2, 3 and 5 transmit the sensed data to the $\mathrm{CH}$ (node 4). Therefore, sensor node 4 only has the role of collecting the sensed data from neighbouring nodes and transmitting the aggregated data to the router node (node 1), while node 1 forwards a single packet to the sink node.

According to results obtained from the first experiment (experiment A) presented in Fig. 8, the router node (node 1) will drain its energy first, because most of its energy is consumed while gathering the sensed data from all nodes in its cluster, in addition to transmitting the 
aggregated data to the sink node. However, when any end-node plays the role of $\mathrm{CH}$ (as in experiment B), the energy consumption of the router node (node 1) will be reduced, as presented in Fig. 9, since node 1 only has the role of forwarding the aggregated packets to the sink node. Therefore, according to the results obtained from the two experiments (A and B), the average energy consumption ratio for a router node is approximately equal to $2 / n_{c}$, whereas the average energy-consumption ratio for an end-node is approximately equal to $1 / n_{c}$, (where $n_{c}$ is the total number of sensor nodes in a cluster -1 ). The sensor node's type $T$ function is presented in Equation 3.

$$
T=\left\{\begin{array}{l}
\frac{1}{n_{c}}, \text { if node is an end }- \text { node } \\
\frac{2}{n_{c}}, \text { if node is router }- \text { node }
\end{array}\right.
$$

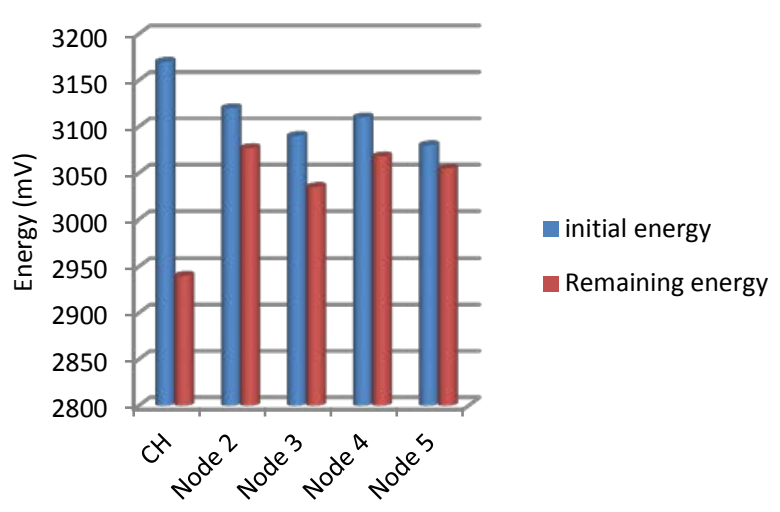

Fig. 8. Estimating the WSN energy consumption in experiment A

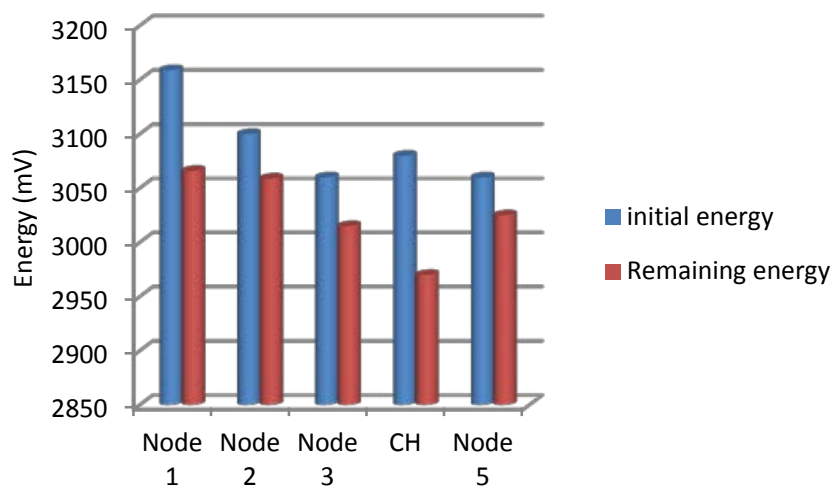

Fig. 9. Estimating the WSN energy consumption in experiment B

The received signal strength function is an indication of the power level received by the receiver radio. The higher the RSS value, the stronger the signal, hence a more reliable and energy-efficient connection. Equation 4 implements the signal strength function $S_{(\mathrm{S}, \mathrm{CH})}$ between sensor node $S$ and a $\mathrm{CH}$.

$$
S_{(s, C H)}=\frac{1}{R S S_{(s, C H)}}
$$


In addition to the clustering approach presented above, the energy consumption is further minimized by reducing the number of packets exchanged through the WSN. The CH has the role of gathering packets from all members and then aggregating the collected packets into a single packet, before transmitting to the sink node. The format of the aggregated packet is depicted in Fig. 10, while Fig. 11 presents the flowchart for the transmission process.

\begin{tabular}{|c|c|c|c|c|c|c|c|c|c|}
\hline Header & MAC Add. & NET Add. & Node ID & Data & Node ID & Data & Node ID & Data & Checksum \\
\hline
\end{tabular}

Fig. 10. Cluster-head packet format

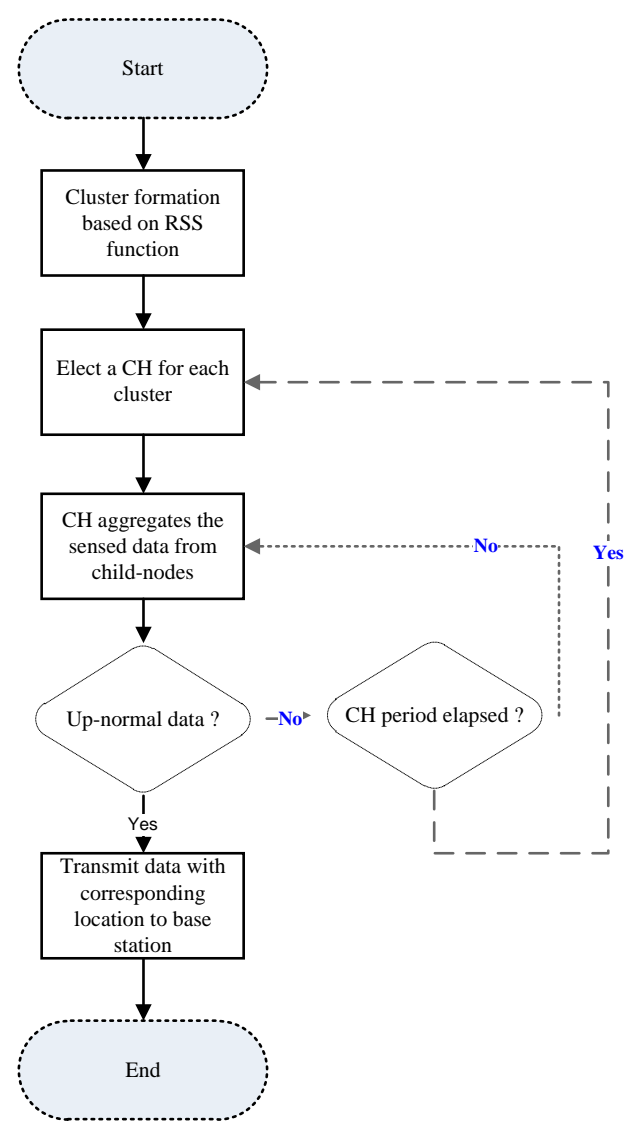

Fig. 11. Flowchart of the transmission process

\section{Experimental Test-bed}

This section presents the experimental test-bed area, in addition to discussing the implementation of sensor-node and network architecture. Fig. 12 shows the experimental test-bed located in the Almoroj area, in the city of Tabuk, Saudi Arabia. The purpose of this deployment was to discover the level of pollution and to estimate the energy consumption for sensor networks employed for environmental monitoring. 


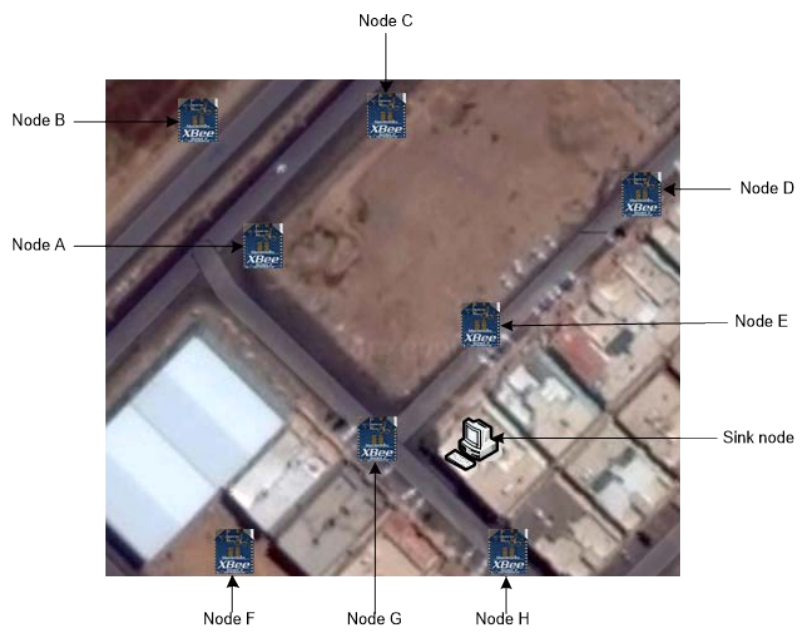

Fig. 12. Deployment of sensor nodes over the geographical area

\subsection{Sensor Node Model}

The experiment test-bed consists of eight sensor nodes distributed over the area of interest. Fig. 13 shows the designed sensor node model, which consists of an XBee series 2 module for communication, an array of sensors and Arduino Pro Mini microcontroller. Firstly, the XBee series 2 module has reliable and effective technical support, in addition to a lower cost than other available modules and an improved power output and data protocol. Secondly, the array of sensors uses three sensors (carbon monoxide, carbon dioxide and temperature) to estimate the level of pollution in a specific area. Thirdly, the Arduino Pro Mini microcontroller has been deployed as a processing subunit, which is based on ATmega328. It is low in cost and energy efficient. Table 2 presents the hardware specification for each device deployed in the experimental test-bed.

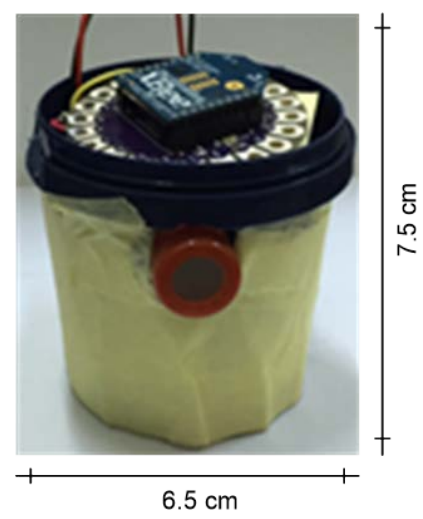

Fig. 13. Sensor node model with on-board sensors 
Table 2. Hardware used in the proposed system

\begin{tabular}{|c|c|c|c|}
\hline & Device & Manufacturer & Power consumption \\
\hline \multirow{3}{*}{ 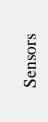 } & CO, MQ-7 & Winsen Electronics Tec. & $\leq 900 \mathrm{~mW}$ \\
\hline & $\mathrm{CO}_{2}, \mathrm{MQ}-2$ & Winsen Electronics Tec. & $\leq 900 \mathrm{~mW}$ \\
\hline & Temp. LM 36 & Sparkfun & $0.165 \mathrm{~mW}$ \\
\hline \multirow{3}{*}{ 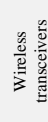 } & XBee series 2 & Digi. & $148.5 \mathrm{~mW}$ \\
\hline & XBee Pro & Digi. & $204 \mathrm{~mW}$ \\
\hline & LilyPad XBee & Sparkfun & - \\
\hline 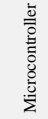 & $\begin{array}{l}\text { Arduino Pro Mini } \\
328-5 \mathrm{~V} / 16 \mathrm{MHz}\end{array}$ & & $96 \mathrm{~mW}$ \\
\hline
\end{tabular}

In order to minimize the total power consumption for environmental monitoring applications, an efficient sampling frequency rate is adopted (20 minutes) and may be adjusted based on the environmental conditions. However, to assure a safe and healthy environment and to detect events which may occur irregularly, the microcontroller needs to be awoken every 2 minutes in order to sense the environment, while the communication module is awoken every 1 minute in order to check if there are any requests coming from neighbouring nodes or the sink node.

\subsection{Network Architecture}

The network architecture is considered in this section. The ZigBee protocol has been deployed as a communication protocol in the experimental test-bed. ZigBee is a low-power, low data-rate, low-cost wireless communication standard, intended to be used in home automation and remote control applications. ZigBee devices are expected to cover 10-75 metres, depending on the RF environment and power output consumption required for a given application. The data rate is $250 \mathrm{kbps}$ at $2.4 \mathrm{GHz}, 40 \mathrm{kbps}$ at $915 \mathrm{MHz}$ and $20 \mathrm{kbps}$ at $868 \mathrm{MHz}$ [37].

The author has employed ZigBee as a communication protocol because the ZigBee protocol was designed to provide easy-to-use wireless data communication characterized by secure and reliable wireless network architecture. The ZigBee protocol utilizes the IEEE 802.15.4 standard as a baseline and adds extra routing and networking functionalities. Compared with the IEEE 802.15.4 network standard, ZigBee has a lower bandwidth, higher latency and asynchronous protocol. In addition, ZigBee is intended to define an application profile that can be shared among different manufactures [38].

ZigBee standard protocol supports different topologies (mesh, star and cluster-tree) and performs three main roles: end-device, router and coordinator. A single coordinator is required for each ZigBee network, where it has a unique personal area identification ID and a channel number, whereas ZigBee router is associated with the multi-hop routing of messages. Finally, ZigBee end-device is an optional network component that is utilized for low-power operations and does not allow association or participation in routing.

In this architecture, two types of devices have been deployed: sensor nodes and sink nodes. The former are stationary nodes (router nodes) distributed over the area of interest, which gather the sensed data from on-board sensors, may enter a sleep mode and may act as a $\mathrm{CH}$. 
The latter are the sink nodes (coordinator nodes), which are responsible for collecting the sensed data from $\mathrm{CHs}$ and display results through a graphical user interface. All the sensor nodes have the same hardware architecture and differ only in the software application. The locations of static sensor nodes are predetermined using Global Positioning System (GPS), and each sensor node's location is stored in a server database system. Table 3 presents experimental test-bed parameters.

Table 3. The experimental test-bed parameters

\begin{tabular}{|l|c|}
\hline \multicolumn{1}{|c|}{ Parameter name } & Value \\
\hline Number of nodes & 8 \\
\hline Average number of hops & 2 \\
\hline Experiment time & 60 minutes \\
\hline Sampling rate & Adaptive \\
\hline Communication protocol & ZigBee \\
\hline Tx energy & $\mathbf{1 . 5} \mathbf{~ m W}$ \\
\hline Rx energy & $\mathbf{1 . 6} \mathbf{~} \mathbf{W}$ \\
\hline Transmission range & 7 metres \\
\hline
\end{tabular}

\section{System Evaluation}

In this work, new ways have been investigated to reduce energy consumption by WSN environmental monitoring applications, with respect to the current state of the art and considering a commercially available, off-the-shelf array of sensors. This section consists of a number of experimental results, at the node and the network levels, used to analyse the network performance.

\subsection{Gas Sensing Results}

In this section, historical data collected from $1^{\text {st }}$ to $30^{\text {th }}$ November 2015 are presented. Measurements collected from eight stationary sensor nodes (labelled A, B, C, D, E, F, G and H). Fig. 14 and Fig. 15 show the $\mathrm{CO}$ and $\mathrm{CO}_{2}$ concentrations, respectively, of three stations over a one-day period. Fig. 16 and Fig. 17 present the $\mathrm{CO}$ and $\mathrm{CO}_{2}$ concentrations, respectively, for all stations over the period of 30 days. Fig. 18 depicts the temperature measurements collected from all stations over the period of one day.

The figures below show the average measurements obtained from all the stations. Fig. 14 shows regular patterns for all days during the month except on Fridays and Saturdays, where the concentrations drop significantly. This is because Friday and Saturday are the two weekend days in Saudi Arabia, so there is less traffic on those days. Since the main source of $\mathrm{CO}$ is the exhaust gas from vehicles, it is expected that the $C O$ concentration would be significantly reduced during the weekends in Tabuk. On the other hand, nodes A, B, and C registered high levels of $\mathrm{CO}$ concentration. This is because they are located close to the main road, where a high number of vehicles pass by. 


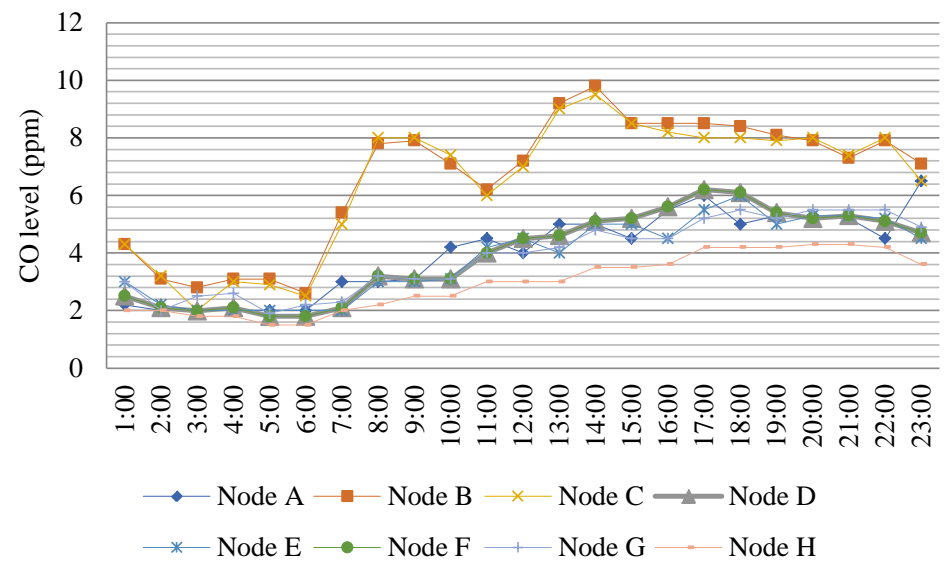

Fig. 14. History of $C O$ level from eight nodes over one-day period

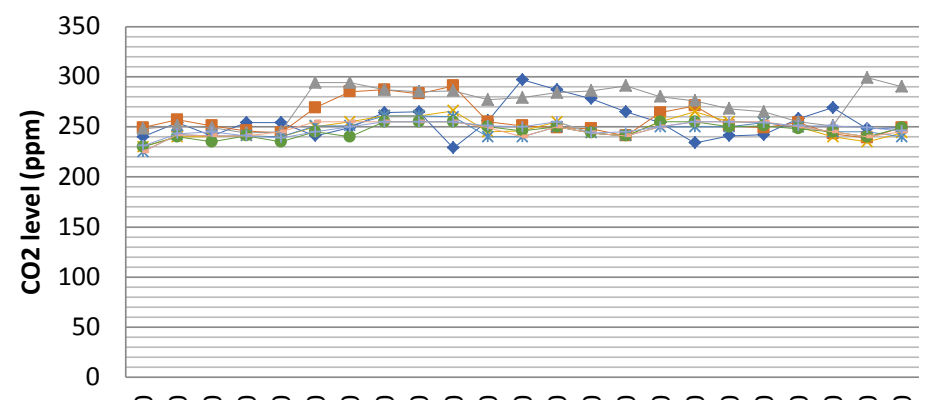

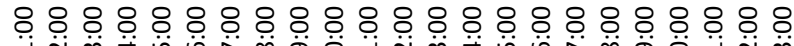

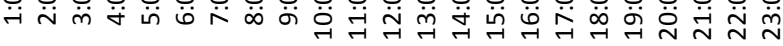

$\longrightarrow$ Node $\mathrm{A} \longrightarrow$ Node B $\longrightarrow$ Node C $\longrightarrow$ Node D

* Node E $\longrightarrow$ Node F $\longrightarrow$ Node G $\longrightarrow$ Node H

Fig. 15. History of $\mathrm{CO}_{2}$ level from eight nodes over one-day period

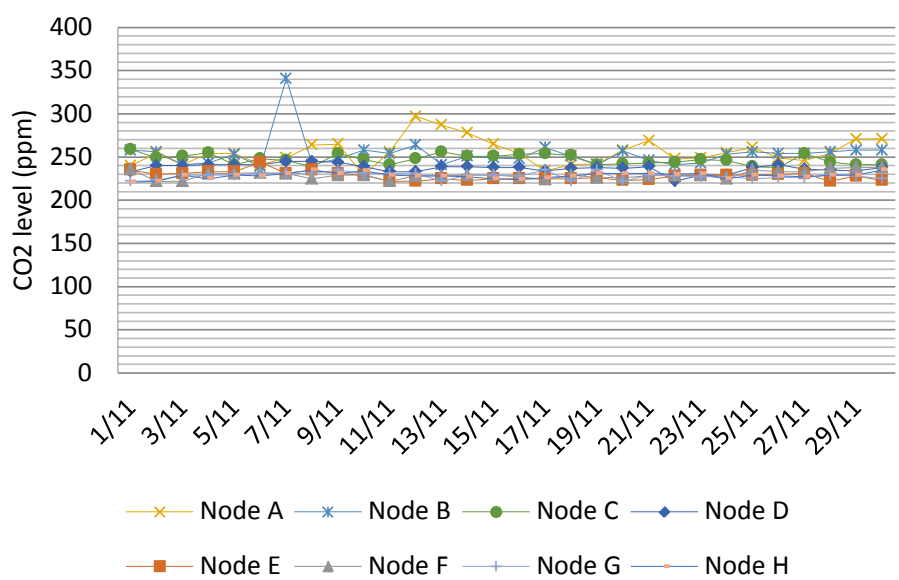

Fig. 16. History of $\mathrm{CO}_{2}$ level from eight nodes over 30-day period 


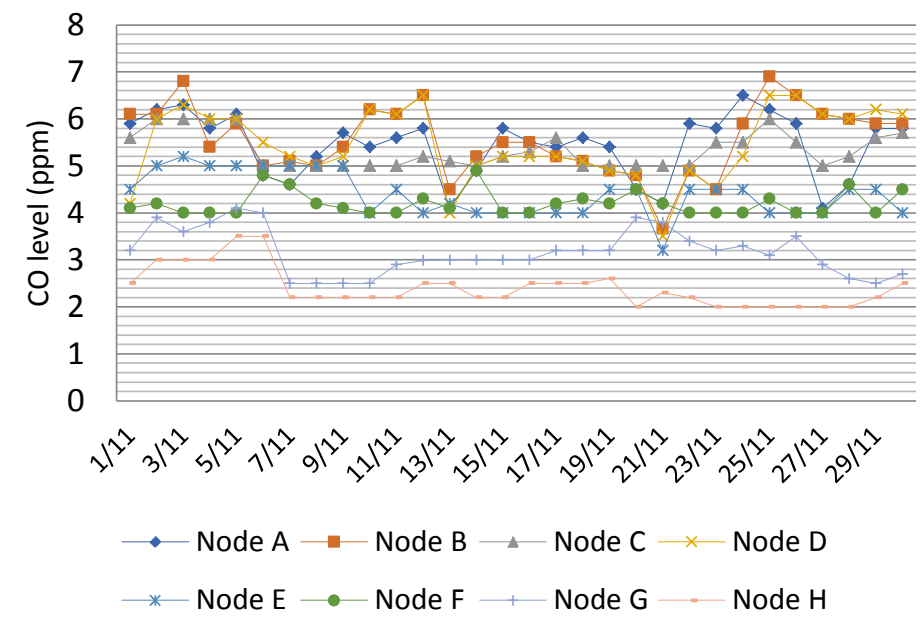

Fig. 17. History of $C O$ level from eight nodes over 30-day period

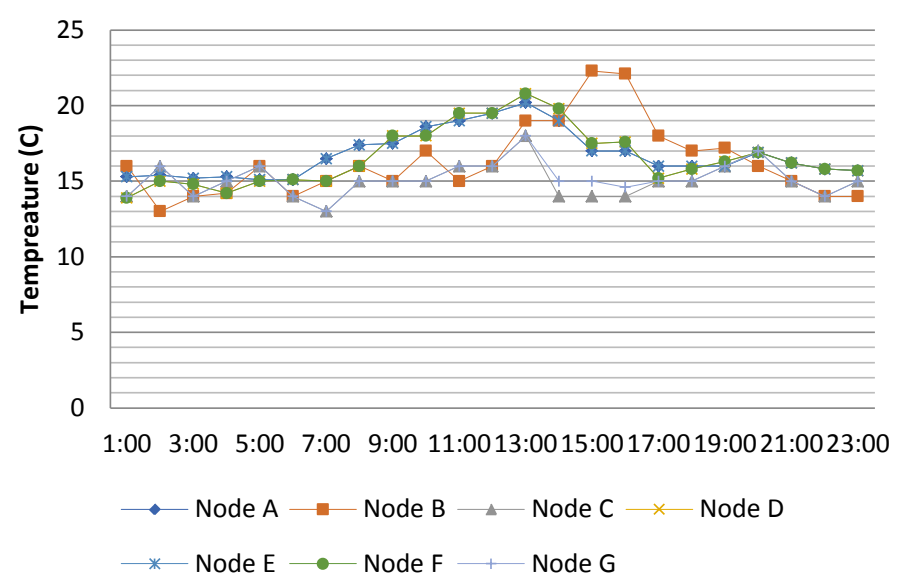

Fig. 18. History of temperature level from eight nodes over one-day period

\subsection{Energy Consumption Estimation Results}

In order to evaluate the energy consumption for the proposed system, three different scenarios were implemented. In Scenario 1, each sensor node transmits its sensed data to the sink node through multi-hop communication, whereas in Scenario 2, LEACH protocol [7] is implemented, where nodes are divided into clusters and each cluster elects a $\mathrm{CH}$. The $\mathrm{CH}$ has the role of gathering sensed data from neighbouring nodes and transmitting them to the sink node. Finally, Scenario 3 implements the proposed system, where a $\mathrm{CH}$ is elected based on the proposed energy function discussed in Section 3.2.

For both Scenarios 2 and 3, two types of messages are exchanged: local and global messages. Local messages are transmitted between cluster members, whereas global messages represent multi-hop data communication between sensor nodes and the sink node. Table $\mathbf{4}$ presents the average energy consumption for each scenario discussed above and the network yield (calculated using Equation 5) for the period of 30 days. 


$$
\text { yield }=\frac{\text { received }_{\text {packets }}}{\text { generated }_{\text {packets }}}
$$

Table 4. Network level performance for 30-day experiment period

\begin{tabular}{|c|c|c|c|}
\hline Scenario & Average energy consumption & Network yield - local & Network yield - global \\
\hline Scenario 1 & $41.6 \%$ & NA & $68.7 \%$ \\
\hline Scenario 2 & $33.9 \%$ & $69.3 \%$ & $71.3 \%$ \\
\hline Scenario 3 & $28.5 \%$ & $68.8 \%$ & $73.5 \%$ \\
\hline
\end{tabular}

Network level results provide a general overview of the network performance, as presented in Table 4. It was noticed that the average network yield for Scenario 1 is about 68.7\%, since each sensor node transmits the collected data from the on-board sensor to the sink node, which may cause delays and traffic congestion, hence increasing the number of dropped packets. In Scenario 2, the network yield was around $71.3 \%$ and in Scenario 3 the network yield was about $73.5 \%$. This is because in both Scenarios 2 and 3 sensor nodes are clustered and a $\mathrm{CH}$ is selected to gather the sensed data from sensor nodes in its cluster and then transmit to the sink node. However, the total number of transmitted packets in Scenario 3 is lower than in Scenario 2, since the proposed clustering function maintains power consumption for all nodes in a certain cluster and minimizes the opportunity to deplete the energy of any node in a cluster, and sensors' data are aggregated before being transmitted to the sink node. Fig. 19 shows the total number of generated local packets and total number of received local packets during our experiment (Scenario 2), whereas the total number of generated global packets and the total number of received global packets for Scenario 3 are presented in Fig. 20.

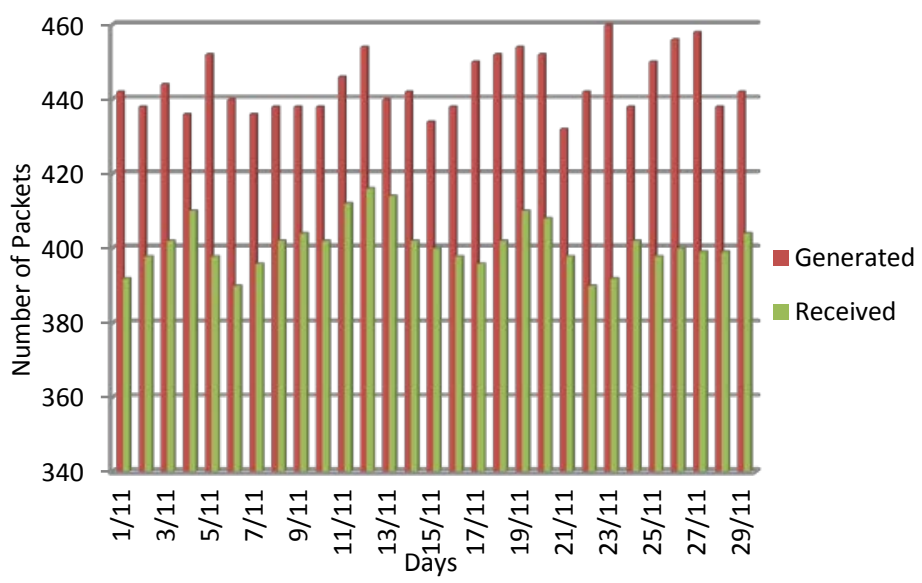

Fig. 19. The network throughput for eight nodes in Scenario 2 


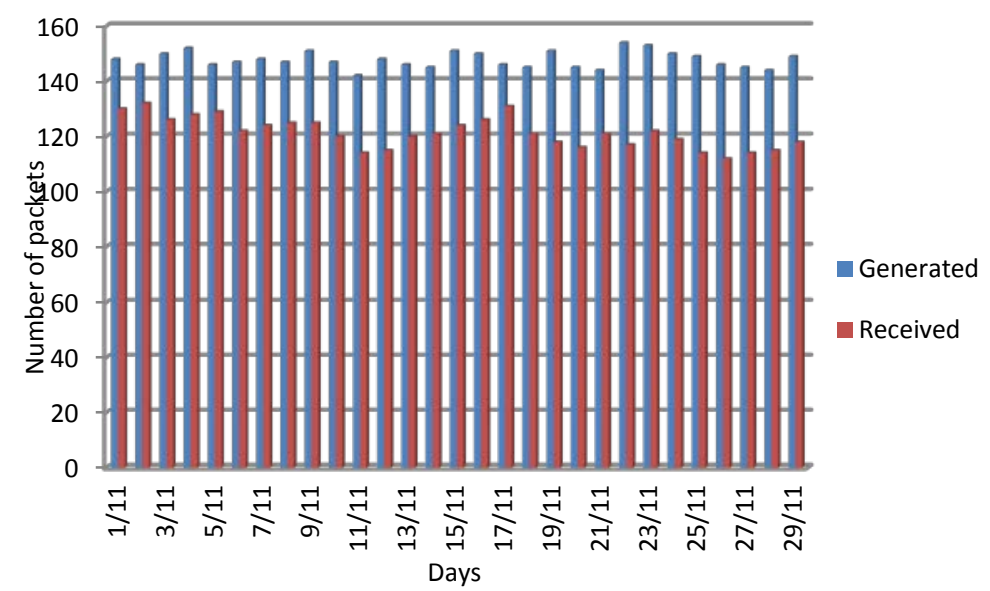

Fig. 20. The network throughput for eight nodes in Scenario 3

Environmental monitoring applications need to run unattended for long time periods. In this section, the energy consumption for the aforementioned three scenarios has been evaluated. Fig. 21 presents the average energy consumption for each sensor node when adopting Scenario 1, where the average energy consumption was around 41.6\%. In Fig. 22, the average energy consumption was estimated when adopting Scenario 2, where the sensor nodes are clustered at the first stage and sensed data are then transmitted to the $\mathrm{CH}$, which aggregates the collected data and transmits a single packet to the sink node. The average energy consumption for Scenario 2 was around 33.9\%.

Fig. 23 presents the average energy consumption for Scenario 3. Sensor nodes are organized into clusters, and then a $\mathrm{CH}$ is selected based on the proposed cluster function, which balances and minimizes the energy for all sensor nodes in a certain cluster. The average energy consumption was around $28.5 \%$ in Scenario 3.

The average energy consumption for all the sensor nodes in the network is shown in Fig. 24. High energy consumption was achieved in Scenario 1, while the best (minimum) energy consumption was accomplished in Scenario 3.

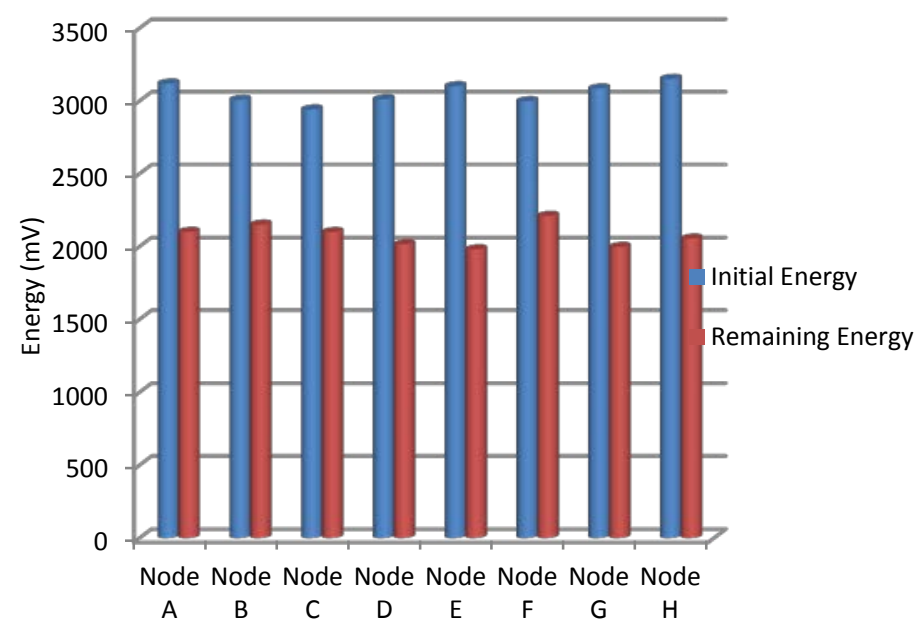

Fig. 21. Estimating the energy consumption for eight nodes using Scenario 1 


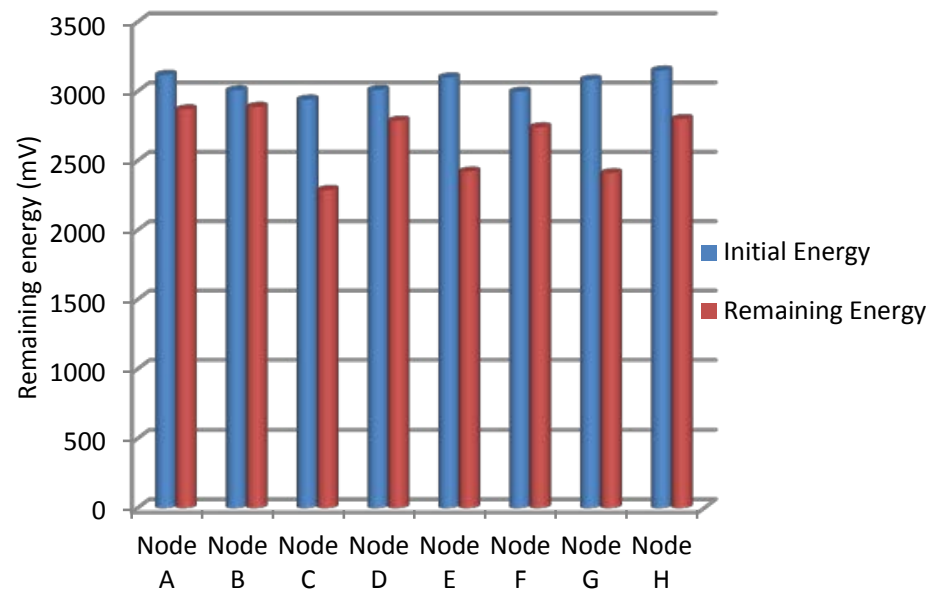

Fig. 22. Estimating the energy consumption for eight nodes using Scenario 2

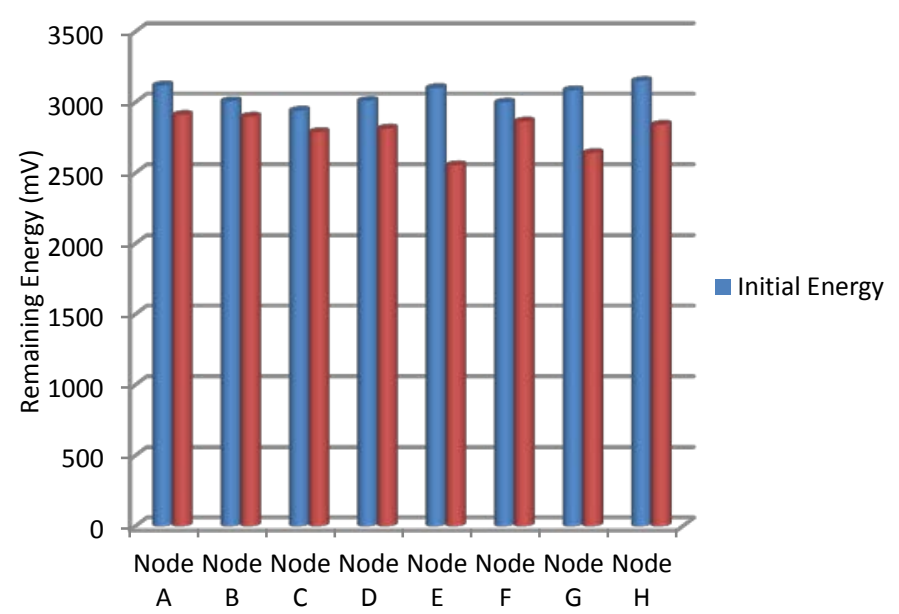

Fig. 23. Estimating the energy consumption for eight nodes using Scenario 3

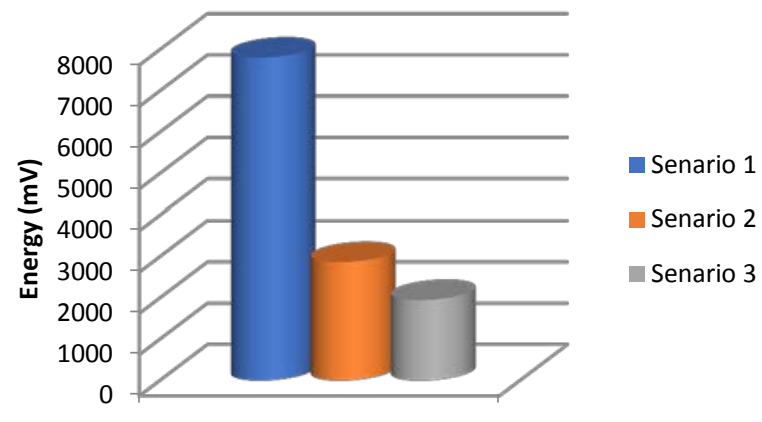

Fig. 24. Total energy consumption in the three scenarios 


\section{Conclusion}

This work addressed the concept of a smart WSN system capable of monitoring outdoor air quality and triggering an alarm in critical situations. The main goal of this approach was the reduction of energy consumption at the node level and the network level. To achieve a longer battery lifetime for WSN in environmental monitoring, an efficient data-gathering system has been investigated for commercially available, off-the-shelf gas sensors. For future work, it will be necessary to deploy a large number of nodes in a wider area in order to test the system's efficiency.

\section{Acknowledgement}

The authors would like to acknowledge financial support for this work from the Deanship of Scientific Research (DSR), University of Tabuk, Tabuk, Saudi Arabia, under grant number S-1436-164.

\section{References}

[1] The United States Environmental Protection Agency (US EPA). Article(CrossRefLink)

[2] T. Alhmiedat, F. Omar, A. Abu Taleb, \& A. Alsswey, "Road Safety and Energy Saving Proposed System: A Zigbee WSN Approach," International Journal of Online Engineering (iJOE), Vol. 11, No. 2. pp. 55-59, 2015. Article(CrossRefLink)

[3] S. Nouha, A. Mellouk, B. Augustin, Y. Amirat, J. Marty, M. Khoussa, A. Abid, \& R. Zitouni, "Wireless Sensor Networks for medical care services," in Proc. of Wireless Communications and Mobile Computing Conference (IWCMC), pp. 571-576, 2011. Article(CrossRefLink)

[4] T. Alhmiedat, "Cyber-Physical Systems," Cyber-Physical Systems: A Computational Perspective, pp. 105-126, 2015. Article(CrossRefLink)

[5] G. Liu, Z. Wang, \& T. Jiang, "QoS-Aware Throughput Maximization in Wireless Powered Underground Sensor Networks," IEEE Transactions on Communications, Vol. 64, No. 11, pp.4776-4789. 2016. Article(CrossRefLink)

[6] T. Alhmiedat, "An Adaptive Energy-Efficient Data Collection System for ZigBee Wireless Sensor Networks," International Journal of Distributed Sensor Networks, 2015. Article(CrossRefLink)

[7] W.R, Heinzelman, A. Chandrakasan, \& H, Balakrishnan, "Energy-efficient communication protocol for wireless microsensor networks," in Proc. of the $33^{\text {rd }}$ annual Hawaii international conference on System sciences. pp. 10. 2000.

[8] J. S. Lee, \& W. L. Cheng, "Fuzzy-logic-based clustering approach for wireless sensor networks using energy predication," IEEE Sensors Journal, Vol, 12. No. 9. pp. 2891-2897. 2012. Article(CrossRefLink)

[9] D. C. Hoang, R. Kumar, \& S. K. Panda, "Fuzzy C-means Clustering protocol for wireless sensor networks," in Proc. of IEEE Int. Symp. Ind. Electron. pp. 3477-3482, 2010. Article(CrossRefLink)

[10] W.B, Heinzelman, A.P. Chandrakasan, \& H, Balakrishnan, "An application-specific protocol architecture for wireless microsensor networks," IEEE Transactions on wireless communications. Vol. 1, No. 4, pp. 660-670. 2002. Article(CrossRefLink)

[11] S. M. Jung, Y. J. Han, \& T. M. Chung. "The concentric clustering scheme for efficient energy consumption," The 9th International Conference on Advanced Communication Technology, Vol. 1, pp. 260-265. 2007. Article(CrossRefLink)

[12] P.G.V., Naranjo, M., Shojafar, H., Mostafaei, Z. Pooranian, \& E, Baccarelli, "P-SEP: a prolong stable election routing algorithm for energy-limited heterogeneous fog-supported wireless sensor networks," The Journal of Supercomputing, pp.1-23. 2016. Article(CrossRefLink)

[13] T. Alhmiedat, "A Survey on Environmental Monitoring Systems using Wireless Sensor Networks," Journal of Networks Vol. 10, No. 11. pp: 606-615. 2016. Article(CrossRefLink) 
[14] M. Rossi, \& D. Brunelli, "Ultra low power wireless gas sensor network for environmental monitoring applications," in Proc. of IEEE Workshop on Environmental Energy and Structural Monitoring Systems (EESMS), pp. 75-81, 2012. Article(CrossRefLink)

[15] J. Baviskar, A. Mulla, A. Baviskar, S. Ashtekar, \& A. Chintawar, "Real time monitoring and control system for green house based on 802.15.4 wireless sensor network," in Proc. of Fourth International Conference on In Communication Systems and Network Technologies (CSNT), pp. 98-103. 2014. Article(CrossRefLink)

[16] S. Bhattacharya, S. Sridevi, \& R. Pitchiah, "Indoor air quality monitoring using wireless sensor network," in Proc. of Sixth International Conference on Sensing Technology (ICST), pp. 422-427. 2012. Article(CrossRefLink)

[17] M. Navarro, T. W. Davis, Y. Liang, \& X. Liang, "A study of long-term WSN deployment for environmental monitoring," in Proc. of IEEE 24th International Symposium on Personal Indoor and Mobile Radio Communications (PIMRC), pp. 2093-2097, 2013. Article(CrossRefLink)

[18] P. Spachos, L. Song, \& D. Hatzinakos, "Prototypes of opportunistic wireless sensor networks supporting indoor air quality monitoring," in Proc. of IEEE Consumer Communications and Networking Conference (CCNC), pp. 851-852. 2013. Article(CrossRefLink)

[19] A. Kadri, E. Yaacoub, M. Mushtaha, \& A Abu-Dayya, "Wireless sensor network for real-time air pollution monitoring," in Proc. of $1^{\text {st }}$ International Conference on Communications, Signal Processing, and their Applications (ICCSPA), pp. 1-5. 2013. Article(CrossRefLink)

[20] V. Jelicic, M. Magno, D. Brunelli, G. Paci, \& L. Benini, "Context-adaptive multimodal wireless sensor network for energy-efficient gas monitoring," IEEE Sensors Journal, Vol. 13, No. 1, pp. 328-338, 2013. Article(CrossRefLink)

[21] G. Barrenetxea, F. Ingelrest, G. Schaefer, M. Vetterli, O. Couach, \& M. Parlange, "Sensorscope: Out-of-the-box environmental monitoring," in Proc. of International Conference on Information Processing in Sensor Networks,(IPSN'08), pp. 332-343. 2008. Article(CrossRefLink)

[22] A. Ghobakhlou, S. Zandi, \& P. Sallis, "Development of environmental monitoring system with wireless sensor networks," in Proc. of $19^{\text {th }}$ International Conference on Modelling and Simulation, Perth, Australia, 2011.

[23] A. Kumar, \& G. P. Hancke, "Energy efficient environment monitoring system based on the IEEE 802.15. 4 standard for low cost requirements," IEEE Sensors Journal, Vol. 14, No. 8, pp. 2557-2566, 2014. Article(CrossRefLink)

[24] S. Saad, A. Shakaff, A. Saad, \& A. Yusof, "Development of wireless sensor network for monitoring indoor air pollutant," in Proc. of International Conference on Mathematics, Engineering and Industrial Applications (ICoMEIA) Vol. 1660, 2015. Article(CrossRefLink)

[25] M. Srbinovska, C. Gavrovski, V. Dimcev, A. Krkoleva, \& V. Borozan, "Environmental parameters monitoring in precision agriculture using wireless sensor networks," Journal of Cleaner Production. Vol. 88, pp. 297-307. 2015. Article(CrossRefLink)

[26] A. Mainwaring, J. Polastre, R. Szewczyk, D. Culler, \& J. Anderson, "Wireless sensor networks for habitat monitoring," in Proc. of the 1st ACM international workshop on Wireless sensor networks and applications, pp. 88-97. 2002. Article(CrossRefLink)

[27] A. Bagula, M. Zennaro, G. Inggs, S. Scott, \& D. Gascon, "Ubiquitous sensor networking for development (usn4d): An application to pollution monitoring," Sensors. Vol. 12, No. 1, pp. 391-414. 2012. Article(CrossRefLink)

[28] M. Gao, F. Zhang, \& J. Tian, "Environmental monitoring system with wireless mesh network based on embedded system," in Proc. of Fifth IEEE International Symposium on Embedded Computing. (SEC'08), pp. 174-179, 2008. Article(CrossRefLink)

[29] S. Saad, L. Kamarudin, K. Kamarudin, W. Nooriman, S. Mamduh, A. Zakaria, A. Shakaff, \& M. Jaafar, "A real-time greenhouse monitoring system for mango with Wireless Sensor Network (WSN)," in Proc. of $2^{\text {nd }}$ International Conference on Electronic Design (ICED), pp. 521-526, 2014. Article(CrossRefLink)

[30] A. Salleh, M. Ismail, R. Mohamad, M. Abd Aziz, M. Othman, \& M. Misran, "Development of greenhouse monitoring using wireless sensor network through ZigBee technology," International Journal of Engineering Science Invention (IJESI) Vol. 2, No. 7, pp. 6-12. 2013. 
[31] M. A. Reddy, \& V. Natarajan, “On-line Monitoring of Green House Gases Storage and Leakage using Wireless Sensor Network,” International Research Journal of Engineering and Technology (IRJET), Vol. 2. No. 1. 2015.

[32] V. Katti, \& A. Pise, "Remote monitoring of Greenhouse Parameters using Zigbee Wireless Sensor Network," in Proc. of International Journal of Engineering Research and Technology. Vol. 3, No. 2, 2014.

[33] O. A. Postolache, J. M. Pereira, \& P. M. B. Girao, "Smart sensors network for air quality monitoring applications," IEEE Transactions on Instrumentation and Measurement, Vol. 58, No. 9, pp. 3253-3262. 2009. Article(CrossRefLink)

[34] A. R. Ali, I. Zualkernan, \& F. Aloul, "A mobile GPRS-sensors array for air pollution monitoring," IEEE Sensors Journal, Vol. 10, No. 10: pp. 1666-1671. 2010. Article(CrossRefLink)

[35] A. Kadri, E. Yaacoub, M. Mushtaha, \& A Abu-Dayya, "Wireless sensor network for real-time air pollution monitoring," in Proc. of 1st International Conference on Communications, Signal Processing, and their Applications (ICCSPA), pp. 1-5, 2013. Article(CrossRefLink)

[36] J. Valente \& W. Pedrycz, “Advances in Fuzzy Clustering and its Applications,” Wiley \& Sons, 2007. Article(CrossRefLink)

[37] T. Alhmiedat, A. Abu Salem, \& A. Abu Taleb, "An improved decentralized approach for tracking multiple mobile targets through ZigBee WSNs," International Journal of Wireless \& Mobile Networks, Vol. 5. No. 3. 2013.Article(CrossRefLink)

[38] S.C, Ergen, “ZigBee/IEEE 802.15. 4 Summary,” UC Berkeley, September, 10, p.17. 2004.

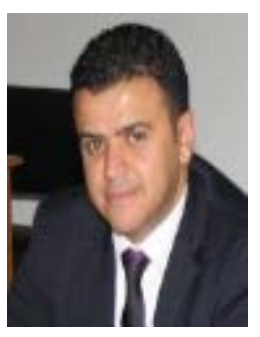

Tareq Alhmiedat is an Assistant Professor in the Department of Information Technology at Tabuk University, Tabuk, Saudi Arabia. He received a Ph.D. in Computer Science from Loughborough University, Loughborough, UK, 2009. MSc in Software Engineering from the University of the West of England, Bristol, UK, 2006, and a BSc degree from Applied Science University, Amman, Jordan, 2004. His main research interests include tracking mobile targets through Wireless Sensor Networks, and Home automation and remote control applications. Dr. Alhmiedat has been involved in various research projects including: SafetyNET, IndoorTrack, and WSN environment monitoring 